\title{
Single-Photon Emission from a Highly Excited CdTe Quantum Dot
}

\author{
S. Nowak, M. Goryca, T. Kazimierczuk, J.A. GaJ, \\ A. Golnik, P. Kossacki \\ Institute of Experimental Physics, University of Warsaw \\ Hoża 69, 00-681 Warszawa, Poland \\ P. Wojnar and G. KarczeWski \\ Institute of Physics, Polish Academy of Sciences \\ al. Lotników 32/46, 02-668 Warszawa, Poland
}

\begin{abstract}
Time-resolved correlation measurements were performed to examine the statistics of photon emission from a single quantum dot at high excitation levels. The range of strong powers was determined by the saturation of intensity of excitonic lines. The significant contribution of pulsed background luminescence forced us to sophisticated analysis of the photoluminescence spectra. For quantum dots grown in the Stranski-Krastanov mode, one-photon emission was found to a good approximation. Fluctuation type quantum dots exhibit a significant level of multiphoton emission.
\end{abstract}

PACS numbers: 73.21.La, 78.55.Et, 78.67.-n, 78.47.-p

\section{Introduction}

Semiconductor quantum dots (QDs) are recognized as promising single-photon sources. The simplest approach in the quest for a single photon-ondemand device is the pulsed optical excitation of a single quantum dot [1]. After excitation with energy higher than the energy gap of the barrier the photocreated carriers or excitons are trapped by the quantum dot. Multiexcitonic states recombine in radiative cascades. Only one photon per cascade is emitted at the wavelength of a single exciton line.

In order to achieve a high probability of photon emission, high excitation levels are required. However, at high excitation a non-vanishing probability of multiphoton emission might be expected. This might occur if the lifetime of photocreated carriers is comparable to the radiative recombination time. Then repeated excitation of the QD might take place.

In particular, an indirect excitation channel - tunneling from higher to lower energy dots - has been also proposed to explain photoluminescence decays 
obtained from ensemble measurements performed on a similar QD system [2]. Such tunneling can lead to delayed excitation of QDs in the low energy part of the spectrum. Therefore it would result in significant probability of many photon emissions following each excitation pulse.

Our study was undertaken to check whether the single photon emission is still dominant even at high excitation power.

\section{Samples and experiment}

We performed measurements on two kinds of samples containing $\mathrm{CdTe} / \mathrm{ZnTe}$ quantum dots. Both samples were grown by molecular beam epitaxy on (001) oriented GaAs substrates.

In the first sample (\#1) a thick CdTe buffer layer was followed by a $700 \mathrm{~nm}$ ZnTe barrier. Then, 6 monolayers of CdTe were grown. Under coverage of amorphous tellurium they formed self-assembled QDs in Stranski-Krastanov transition. After tellurium desorption the structure was covered by a $100 \mathrm{~nm} \mathrm{ZnTe}$ cap layer. Transmission electron microscopy measurements performed on similar samples without a cap layer revealed quantum dots of a density of $10^{10} \mathrm{~cm}^{-2}[3]$.

The second sample (\#2) has a similar structure. The CdTe buffer layer was overgrown by a $100 \mathrm{~nm} \mathrm{ZnTe}$ barrier. Then two monolayers of CdTe were grown, forming fluctuation-type quantum dots. Dots formation was achieved in situ annealing under $\mathrm{Cd}$ atom beam. The quantum dot layer was covered by a $50 \mathrm{~nm}$ ZnTe barrier. The dots density measured by transmission electron microscopy on samples without a ZnTe cap layer revealed dots density of $10^{12} \mathrm{~cm}^{-2}$ [4].

The difference between the two samples quantum dots density is of two orders of magnitude. The QDs differ also in the size and depth of the confining potential well. As a result self-assembled QDs have deeper potential, containing many excitonic states. On the contrary, fluctuation-type quantum dots have shallower confining potential and the biexciton is assumed to be their highest excitonic state [4].

We performed measurements at $T=1.8 \mathrm{~K}$ in a microluminescence setup used previously in [5]. The light spot diameter on the surface of the sample was of $0.5 \mu \mathrm{m}$. The QDs were excited by a femtosecond pulsed $\mathrm{Al}_{2} \mathrm{O}_{3}$ :Ti laser with frequency doubling. The pulses were shorter than $0.4 \mathrm{ps}$, their repetition rate was $76 \mathrm{MHz}$ and central wavelength $400 \mathrm{~nm}$. Photoluminescence spectra for single QD emission lines and photon correlations were measured at different average excitation power. Photon correlation measurements were performed in a Hanbury-Brown and Twiss detection scheme [6] with two avalanche photodiodes as detectors. Photon correlations were measured at emission line related to neutral exciton recombination.

The photoluminescence lines related to the measured single QDs were separated from the long wavelength tail of the broad photoluminescence. Thus we studied mainly QDs of relatively low confinement energy and thus longer sizes. 


\section{Results}

\subsection{Microphotoluminescence}

The photoluminescence measurements for single QDs were performed for different excitation powers. Typical spectra and the power dependence of the intensity of selected lines for the sample \#1 are presented in Fig. 1. The lines were previously identified in [7].
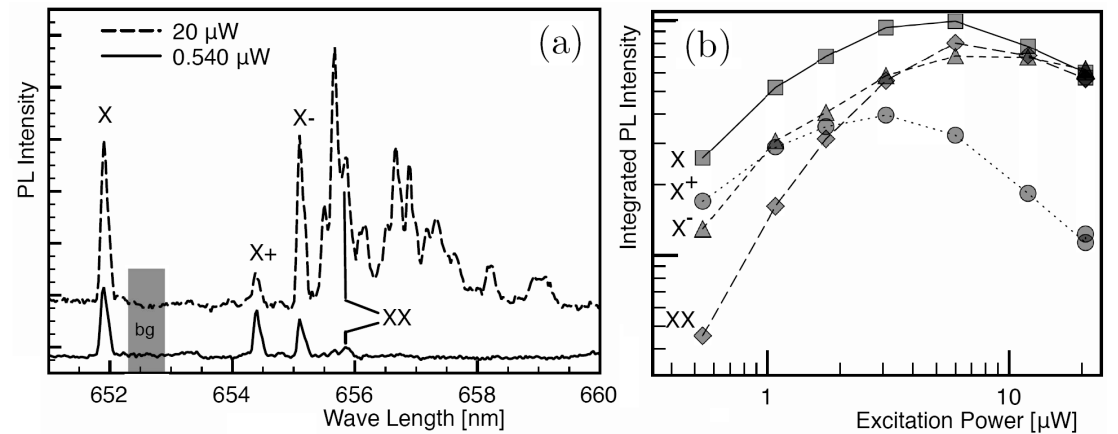

Fig. 1. (a) Microphotoluminescence spectra of a selected deep QD (sample \#1) under pulsed excitation above the energy gap of the barrier at two indicated excitation power values. The gray rectangular denotes the region where the background was measured. (b) Excitation power dependence of excitonic line intensities of a selected deep QD (sample \#1).

At low excitation power the spectrum is dominated by lines related to the neutral exciton $(\mathrm{X})$ and negatively and positively charged excitons $\left(\mathrm{X}^{-}\right.$and $\left.\mathrm{X}^{+}\right)$. When excitation power is increased $\mathrm{X}, \mathrm{X}^{-}$and $\mathrm{X}^{+}$tend to saturate and higher complexes become visible. Additionally, a shift of the average charge state of the QD toward negative states is visible. It is seen primarily as a relative increase in intensity of $\mathrm{X}^{-}$line with respect to $\mathrm{X}^{+}$line.

At high excitation power a spectrally broad background appears. This luminescence is pulsed as the excitation but negligibly depends on the position of the excitation spot on the sample.

The behavior of shallow QDs selected from sample \#2 is very similar except of the absence of multiexciton lines.

The saturation of the single exciton lines $\left(\mathrm{X}, \mathrm{X}^{-}\right.$and $\left.\mathrm{X}^{+}\right)$was expected. For a $\mathrm{QD}$ that emits no more than one cascade per excitation pulse, the $\mathrm{X}$ line intensity should saturate as the number of $\mathrm{X}$ photons should not exceed the number of laser pulses.

\section{2. $X-X$ autocorrelation}

The autocorrelation measurements were performed in order to test whether the $\mathrm{X}$ emission has a single photon character. We used the technique applied in 


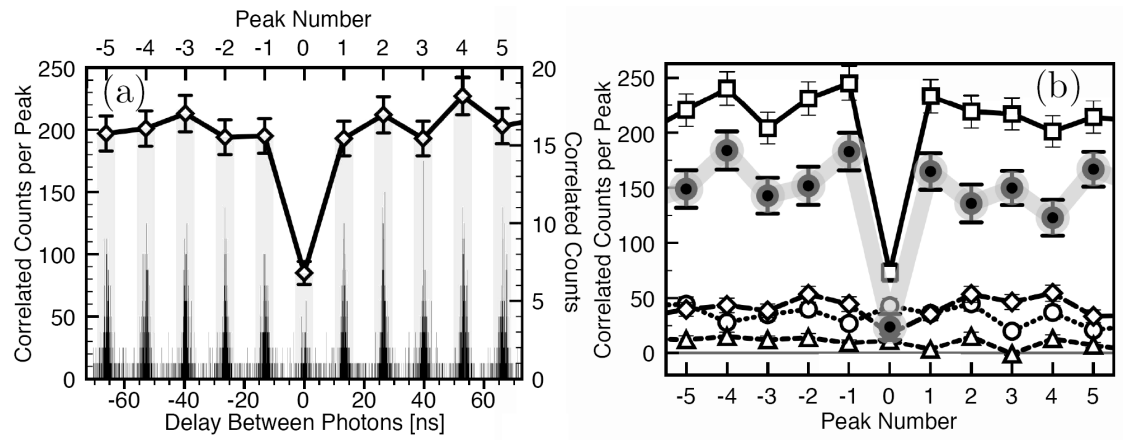

Fig. 2. (a) Example histogram of X-X correlation counts versus delay: black - general histogram with time bin of $73.4 \mathrm{ps}$, diamond points - counts summed over particular peaks (shaded areas), corrected for dark counts. (b) Histograms summed over particular peaks versus peak numbers measured in four correlation measurements at excitation power of $20 \mu \mathrm{W}$ : $\mathrm{X}-\mathrm{X}$ (squares), $\mathrm{X}-\mathrm{bg}$ (diamonds), bg-X (circles) and bg-bg (triangles). Gray points and curve represent background-corrected $\mathrm{X}-\mathrm{X}$ correlation.

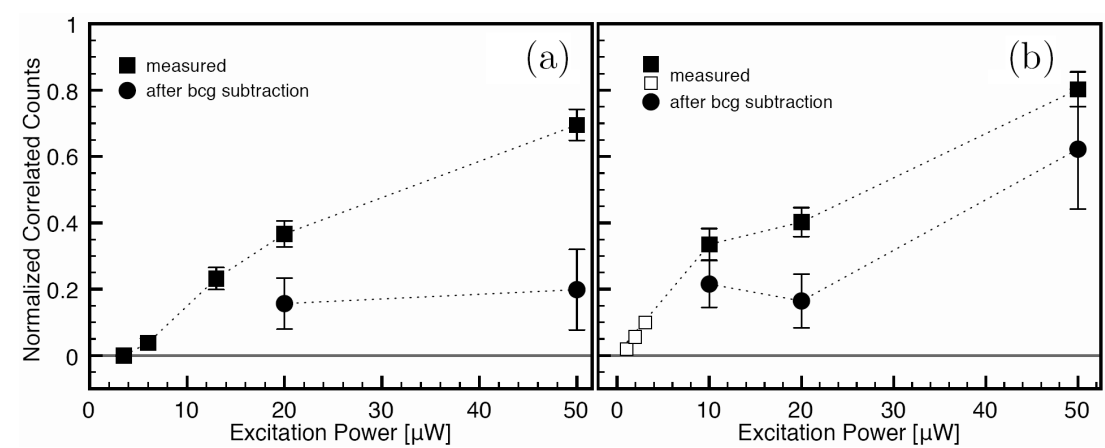

Fig. 3. Correlated counts summed over the zero-delay peak versus excitation power: as measured (squares) and after background correction (circles); (a) sample \#1 (StranskiKrastanov QDs); (b) sample \#2 (fluctuation type QDs). Blank squares are taken from [8].

[5] for low excitation levels. In this work correlation measurements were done well above the saturation power. An example data set is presented in Fig. 2.

A pronounced periodical peak structure, related to laser pulses, is visible in Fig. 2a. Far from the peaks (outside of shaded regions in Fig. 2) correlated signal originates from dark counts. The dark count contribution has been subtracted for further analysis. The numbers of counts thus obtained, summarised for each peak, are shown in Fig. 2b.

For each excitation power an antibunching dip is observed around zero delay time. The counts at zero delay time correspond to the detection of two photons following the same pulse. The single photon character of the emission is demon- 
strated by the appearance of the antibunching dip.

However, the antibunching dip becomes less pronounced with increasing the excitation power. This may lead to the conclusion that multi-photon emission following a single excitation pulse becomes highly probable.

In order to analyze the data correctly one has to take into account also the correlation events with photons which originate not from the selected quantum dot emission but from the background luminescence. As presented in Sect. 3.1 and in Fig. 1, a significant background is present in the photoluminescence spectra measured at high excitation. Thus the measured correlation signal consists of four components: pure $\mathrm{X}-\mathrm{X}$ correlation, $\mathrm{X}$-background correlation, background-X correlation, and background-background correlation. Pure $\mathrm{X}-\mathrm{X}$ correlation can be obtained by subtracting the background components from the measured correlation.

Therefore we measured the cross-correlation of the excitonic line and the background, the cross-correlation of the background and the excitonic line, and the autocorrelation of the background. The measurement of both cross-correlations was necessary as the detection efficiency of both channels was different. The background was measured at the flat part of the excitonic spectrum, close to the excitonic line (see the gray rectangle in Fig. 1).

As shown in Fig.3, after subtraction of the additional correlations the antibunching dip becomes much deeper for both samples at most excitation powers. The zero-delay values are close to zero for the Stranski-Krastanov sample (\#1), indicating that one-photon emission occurs to a good approximation even at high excitation level (see Fig. 3a). The sample with fluctuation-type QDs (\#2) exhibits significant departure from the one-photon emission model (Fig. 3b). Inter-dot excitation transfer [2] in this sample may be the cause of the observed events of second photon emission. The high QDs density of sample \#2 makes such excitation transfer probable.

\section{Conclusions}

We demonstrated single photon emission of the Stranski-Krastanov QDs even at high excitation power.

The qualitative analysis of the QD luminescence at high excitation power indicates a significant contribution of the pulsed background in time-resolved measurements. For the possible application a reduction of the background luminescence is necessary. Therefore further studies on the nature of the pulsed luminescence background are required.

\section{Acknowledgments}

This work was supported by grants of Polish Ministry of Science and Higher Education financed in 2006-2010 and by European project No. MTKD-CT-2005029671. 


\section{References}

[1] P. Michler, A. Kiraz, C. Becher, W.V. Schoenfeld, P.M. Petroff, Lidong Zhang, E. Hu, A. Imamoglu, Science 290, 2282 (2000).

[2] K.P. Korona, P. Wojnar, J.A. Gaj, G. Karczewski, J. Kossut, J. Kuhl, Solid State Commun. 133, 369 (2005).

[3] F. Tinjod, B. Gilles, S. Moehl, K. Kheng, H. Marietteb, Appl. Phys. Lett. 82, 24 (2003).

[4] G. Karczewski, S. Maćkowski, M. Kutrowski, T. Wojtowicz, J. Kossut, Appl. Phys. Lett. 74, 20 (1999).

[5] J. Suffczyński, T. Kazimierczuk, M. Goryca, B. Piechal, A. Trajnerowicz, K. Kowalik, P. Kossacki, A. Golnik, K.P. Korona, M. Nawrocki, J.A. Gaj, G. Karczewski, Phys. Rev. B 74, 085319 (2006).

[6] R. Hanbury-Brown, R.Q. Twiss, Nature 178, 14491450 (1956).

[7] K. Kowalik, O. Krebs, A. Golnik, J. Suffczyński, P. Wojnar, J. Kossut, J.A. Gaj, P. Voisin, Phys. Rev. B 75, 195340 (2007).

[8] J. Suffczyński, Ph.D. Thesis, University of Warsaw, Warsaw 2007, p. 75. 